\title{
TWO-LAYER FINITE-DIFFERENCE SCHEMES FOR THE KORTEWEG-DE VRIES EQUATION IN EULER VARIABLES
}

\author{
V.I. MAZHUKIN*, A.V. SHAPRANOV, E.N. BYKOVSKAYA \\ Keldysh Institute of Applied Mathematics of the RAS, Moscow, Russia. \\ * Corresponding author. E-mail: vim@modhef.ru
}

\section{DOI: 10.20948/mathmontis-2020-49-5}

Summary. A family of weighted two-layer finite-difference schemes is presented. Using the example of the numerical solution of model problems on the propagation of a single soliton and the interaction of two solitons, the high quality of explicit-implicit schemes of the CrankNichols type with the parameter $\sigma=0.5$ and the order of approximation $O\left(\Delta t^{2}+\Delta x^{2}\right)$ is shown. Completely implicit two-layer difference schemes with the parameter $\sigma=1$ and $\mathrm{O}(\Delta t$ $+\Delta x^{2}$ ) are characterized by absolute stability with a low solution accuracy due to a high approximation error. The family of explicitly implicit difference schemes is absolutely unstable if the explicitness parameter $\sigma<0.5$ prevails. Analysis of the structure of the approximation error, performed using the modified equation method, confirmed the results of numerical simulation.

\section{INTRODUCTION}

The theory of nonlinear waves was originally associated with the study of problems in gas and hydrodynamics, which include a number of varied and striking problems of applied and fundamental nature [1], which lead to the need to analyze the huge and growing data associated with multidimensional nonlinear dynamics.

Initially, the Korteweg de Vries equation (KdV) arose from the needs of hydrodynamics [2], [3] associated with the propagation of nonlinear solitary waves in shallow water $[4,5]$, which ended with the discovery of solitons [6]. The KdV equation was the first nonlinear wave equation to have soliton solutions. Note that the discovery of solitons [6] was carried out on the basis of a computational experiment. As it turned out, solitons, which are stable formations, have a number of amazing properties. Thus, the propagation of a soliton in the form of a nonlinear solitary wave allows it to maintain its shape and speed during its motion. In addition, solitons are characterized by elastic interaction with each other. In the course of a collision, they first deform and then restore their original parameters and their original shape. Taking into account that the propagation of a soliton is described by a nonlinear equation, then the principle of superposition, as it is understood in linear systems, according to which the sum of particular solutions is also a solution, does not hold for it. Solitons exactly interact with each other, first deforming, and then, restoring their original parameters, in contrast to linear solution systems, which pass through each other. The only result of the interaction of solitons may be some phase shift. This confirms that solitons are precisely nonlinear solutions.

Due to the rapid development of high-performance computing technology, computational algorithms and methods of modern mathematical modeling, it became possible to study more

2010 Mathematics Subject Classification: 01-08, 35Q53, 35C08.

Key words and Phrases: Two-layer finite-difference schemes, Korteweg-de Vries equation, Euler variables, Soliton solutions. 
and more complex problems of hydrodynamics [7], nonlinear optics [8], plasma physics [9, 10] and solids [11]. However, since the complexity of the problems under study is ahead of the development of the used computer technology, the problem of increasing the efficiency of mathematical methods and approaches remains relevant.

The active use of solitons in the study and solution of nonlinear wave equations [12] describing physical phenomena in many areas [13] stimulated interest in methods for solving the $\mathrm{KdV}$ equation. The $\mathrm{KdV}$ equation was solved numerically by various methods, such as the Galerkin method [14-16], the collocation method [17, 18], the finite element method [19-21], the finite-difference method [22-30], etc. The choice of one or another numerical solution method largely determines the quality of numerical modeling. It should also be kept in mind that it is far from being indifferent, at the expense of what costs the final result of modeling is achieved. Therefore, it is quite natural to impose on computational algorithms the requirement not only of stability and efficiency, but also of simplicity of implementation. Finite-difference methods possess the greatest combination of these properties.

Earlier, in [24, 25], the results of the analysis of two-layer difference schemes for the KdV equation from the point of view of integral conservation laws were reported. The concept of $L_{2}$-conservatism of a difference scheme was used as the ability of its solution to satisfy the grid analogue of conservation laws $[31,32]$. The $L_{2}$-conservatism principle makes it possible, when constructing efficient algorithms, to ensure that they satisfy the grid analogs of the basic properties of the differential problem.

Based on the $L_{2}$-conservatism principle for the Korteweg-de Vries equation, it was shown that explicit two-layer difference schemes do not satisfy the $L_{2}$-conservatism condition and, moreover, are absolutely unstable even in the weakest $L_{2}$ norm. In the same papers, this principle was applied to construct a family of three-layer completely conservative (conservative and $L_{2}$-conservative) weighted difference schemes.

In this paper, we numerically and analytically study a family of two-layer difference schemes for the KdV equation, which includes both explicit and implicit schemes.

\section{STATEMENT OF THE PROBLEM}

$\mathrm{KdV}$ equation in the divergence form

$$
\frac{\partial u}{\partial t}+\alpha \frac{\partial}{\partial x}\left(\frac{u^{2}}{2}\right)+\beta \frac{\partial^{3} u}{\partial x^{3}}=0
$$

includes nonlinear and dispersion terms, the competition of which determines the behavior of the solution. The solution of equation (1) is represented in the form of a moving soliton.

The soliton is a stationary unipolar pulse traveling in the positive direction of the $\mathrm{X}$ axis with a speed Q

$$
u(x, t)=\frac{A}{\operatorname{ch}\left[\left(x-x_{0}-Q t\right) / \delta\right]^{2}},
$$

where $A=3 Q / \alpha$ is the amplitude and $\delta=\sqrt{4 \beta / Q}$ is the half-width (at the level of $0.42 A$ ) of the soliton. The analytical representation of the soliton will be used to test the computational method. 
To complete the formulation of the Cauchy problem, it is necessary to set the boundary conditions. As the initial condition, the grid representation of the soliton (2) is specified. Equation (1) requires the setting of three boundary conditions on two boundaries of the final computational domain.

At the left boundary: $\left.\frac{\partial^{2} u}{\partial x^{2}}\right|_{x=0}=0, \quad$ At the right boundary $\left.u\right|_{x=L}=0,\left.\quad \frac{\partial u}{\partial x}\right|_{x=L}=0$.

\section{FINITE-DIFFERENCE APPROXIMATION}

In the space of variables $\Omega_{(x, t)}$, we construct a difference grid uniform in $\mathrm{x}$

$$
\omega_{\Delta x}^{\Delta t}=\left\{\left(x_{m}, t^{k}\right): x_{m+1}=x_{m}+\Delta x, t^{k+1}=t^{k}+\Delta t^{k}, \quad m=0, \ldots, M, k=0, \ldots, K\right\},
$$

on which the grid function is defined $u_{m}^{k} \equiv u\left(x_{m}, t^{k}\right)$.

(a)

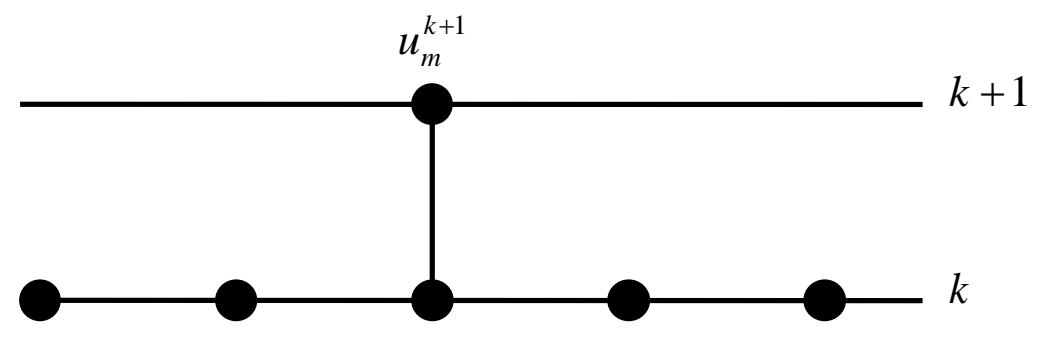

a)

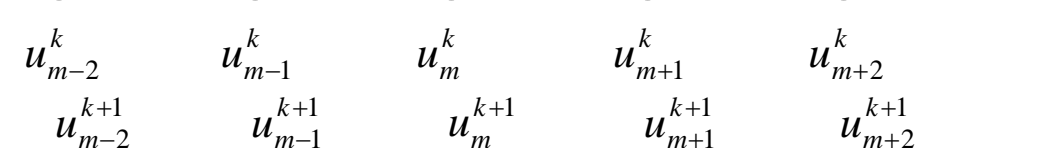

(b)
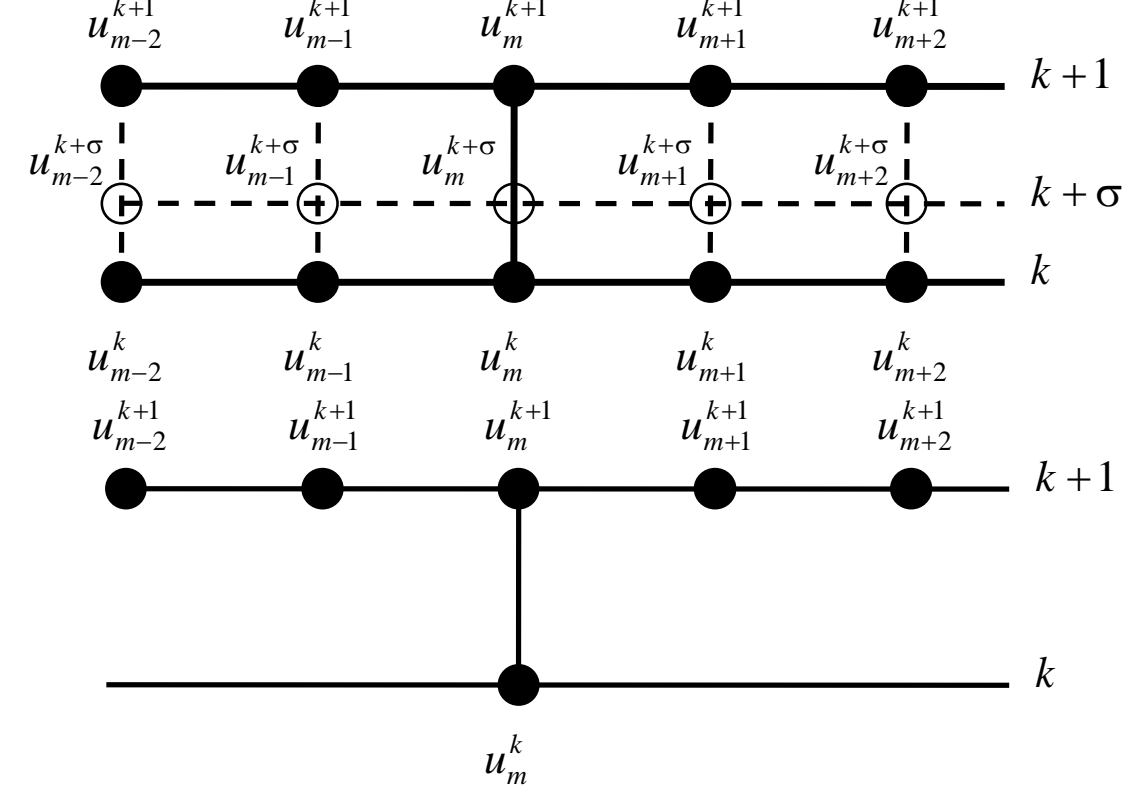

Fig. 1. Grid patterns, implemented by the scheme (5), for different values of $\sigma$. (a) $\sigma=0$, i.e. $u^{k+\sigma}=u^{k}$ (explicit scheme); (b) $0<\sigma<1$, empty circles are fictional nodes at the intermediate time layer $(k+\sigma)$; (c) $\sigma=1$, i.e. $u^{k+\sigma}=u^{k+1}$.

Using (4), we construct a family of finite-difference schemes for the approximation of the equation (1): 
$\frac{u_{m}^{k+1}-u_{m}^{k}}{\Delta t}+\frac{\alpha}{\Delta x}\left(\frac{\left(u_{m+1}^{k+\sigma}+u_{m}^{k+\sigma}\right)^{2}}{8}-\frac{\left(u_{m}^{k+\sigma}+u_{m-1}^{k+\sigma}\right)^{2}}{8}\right)+\beta \frac{u_{m+2}^{k+\sigma}-2 u_{m+1}^{k+\sigma}+2 u_{m-1}^{k+\sigma}-u_{m-2}^{k+\sigma}}{2 \Delta x^{3}}=0$

where $u_{m}^{k+\sigma}=(1-\sigma) u_{m}^{k}+\sigma u_{m}^{k+1}, \sigma$ is the weight coefficient, which values $\sigma \epsilon[0-1]$ determine the degree of "implicitness" of the difference scheme. The value $\sigma=0$ corresponds to a completely explcit scheme, while $\sigma=1$ - to a completely implicit one.

In all finite-difference schemes (5), the second order of approximation in spatial $\mathrm{x}$ is implemented for the derivatives of both the 1 st and the $3 \mathrm{rd}$ order. With respect to the time variable $\mathrm{t}$ for all values $\sigma \neq 0.5$, the schemes (5) have the 1 st order of approximation. For, $\sigma=$ 0.5 , the expression (5) is an implicit difference scheme of the Crank-Nichols type with the second order of approximation in time. Fig. 1. shows grid patterns for different values of $\sigma$.

\section{COMPUTATIONAL ALGORITHM}

The difference approximation (5), applied to the interior points of the computational domain, generates a system of nonlinear equations with respect to quantities $\tilde{u}$ on a new time layer. This system is solved at each time step by the Newton iterative method, for which the procedure of its linearization is performed, after which it is transformed into a linear system of equations with a 5-diagonal (band) matrix. In the only case of $\sigma=0$, the matrix degenerates into the identity one, and the scheme becomes explicit.

\section{COMPUTATIONAL EXPERIMENT}

For numerical testing, the following parameters of the equation $\alpha=6, \beta=1$ and soliton (2) presented in Fig. 2(a) were used: $x_{0}=10, Q=4 \Rightarrow A=2, \delta=1$;

In the computational domain with the size $L=420$, a computational grid was constructed containing $M=2100$ intervals with a spatial step size $\Delta x=0.2$. The total width of the soliton $2 \delta$ contained 10 intervals.

For implicit schemes $\sigma \neq 0$, a mechanism for automatic time step selection was implemented, based on the following parameters: the maximum allowable number of iterations at each time step was 3-4, the criterion of convergence of the iterative process includes the relative and absolute errors, the values of which were taken equal to $10^{-9}$. For an explicit scheme $(\sigma=0)$, the time step is discussed below.

Figure 2(b) shows the solution using the Crank-Nichols-type scheme $(\sigma=0.5)$ at the time $t=100$ when the soliton has moved from the initial position to a distance of $400 \delta$. The transfer speed determined from the numerical solution turned out to be $1.1 \%$ less than the analytical value (i.e., the lag was about $4 \delta$ ). In this case, the amplitude of the soliton fluctuates around the average value with a standard deviation of $0.3 \%$, and the average value itself is only $0.011 \%$ greater than the analytical one (Fig. 4(a)). That is, it can be assumed that the numerical solution preserves the amplitude of the soliton with good accuracy during the entire calculation process.

The time step during the entire computational process fluctuated with a small amplitude around a constant value (Fig. 4(b)). These small fluctuations were associated with the organization of the automatic step selection mechanism. 
(a)

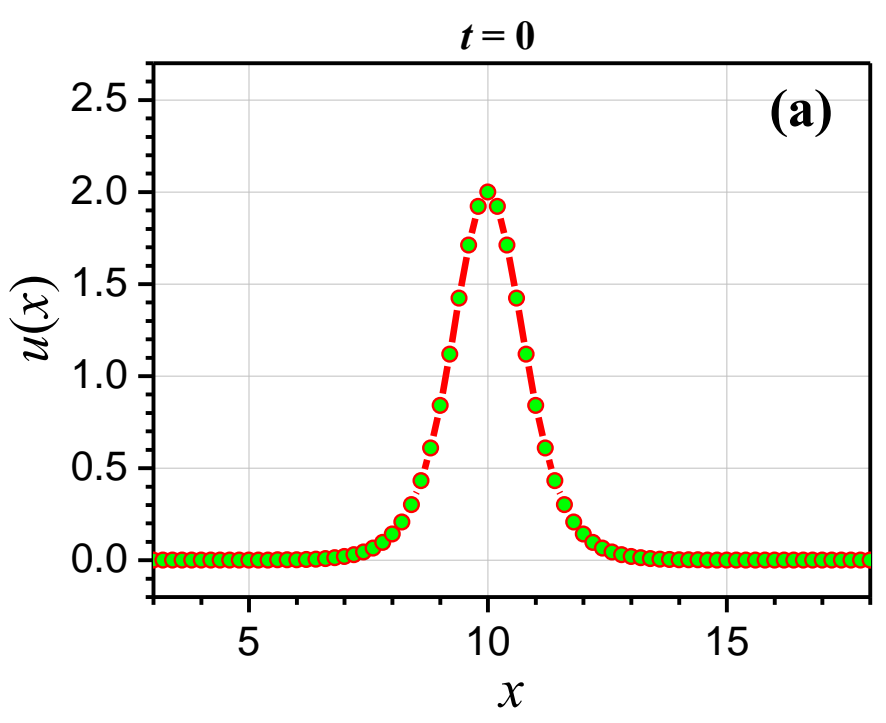

Fig.2 (a). Spatial profile of soliton at the initial time $\mathrm{t}=0$.

(b)

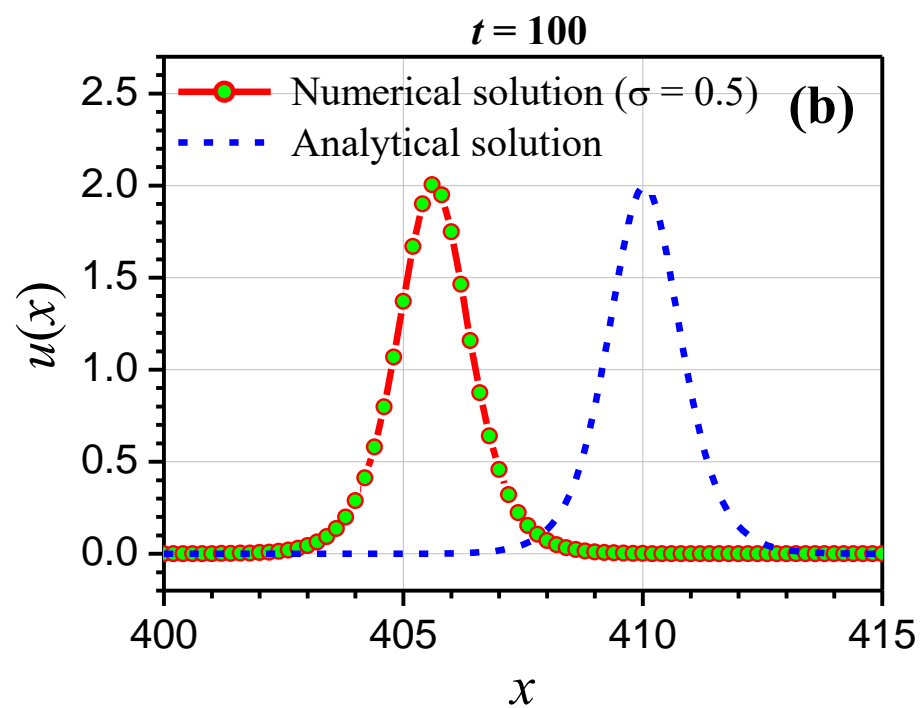

Fig.2 (b). Comparison of the numerical solution obtained using a scheme of Crank-Nichols type $(\sigma=0.5)$ with the analytical one at the time $\mathrm{t}=100$.

The solution using a completely implicit scheme $(\sigma=1)$ leads to significantly worse results (Fig. 3). This is due to the high schematic viscosity, which in this case is the reason for a strong drop in the amplitude of the soliton with time, which in turn decreases its velocity (Fig. 4(a)). In this case, as the amplitude decreases, the integration step increases (Fig.4(b)). However, at the initial moments of time, when the amplitude is not yet very different from the initial value, the time step turns out to be about 2 times less than for the Crank-Nichols type scheme.

Figure 5 shows a numerical solution using an explicit difference scheme $(\sigma=0)$. Figure (a) shows the solution with a time step $\Delta t=0.0001$. By the moment of time $t=3$, a loss of stability occurs and further calculation becomes impossible. Note that a decrease in the integration step pushes further in time the moment of stability loss. 


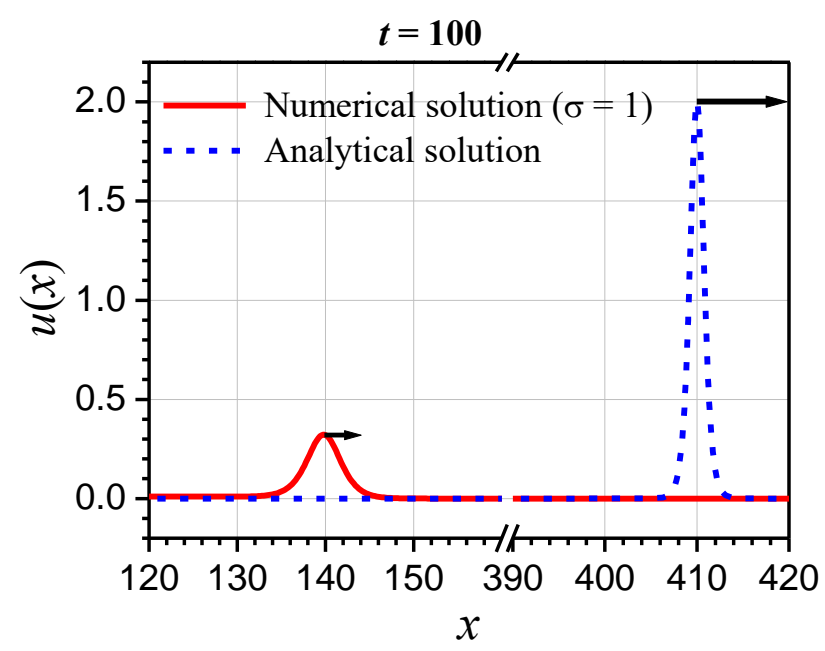

Fig.3. Comparison of numerical solution using a completely implicit scheme $(\sigma=1)$ with the analytical one at the time $\mathrm{t}=100$.

(a)
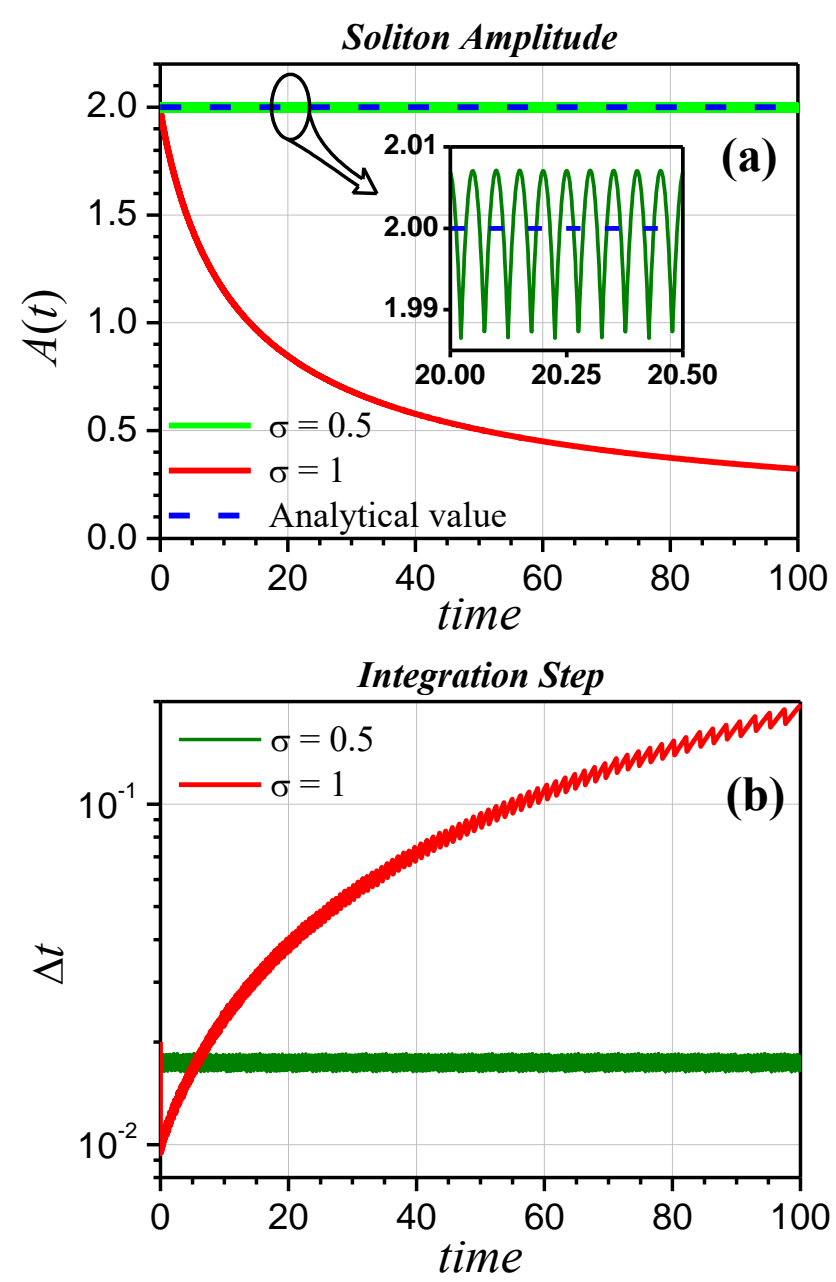

Fig.4. The soliton amplitude versus time (a) and automatically chosen integration step (b) for two implicit schemes: $\sigma=0.5$ and 1. 
Thus, decreasing the step by a factor of 2 to $\Delta t=5 \cdot 10^{-5}$ leads to the fact that the destruction of the solution occurs at about time $t=6$. Figure (b) shows the loss stability at the time of $t=30$ when integrating with a step $\Delta t=1 \cdot 10^{-5}$. According to the results obtained in theoretical works $[24,25]$, explicit two-layer difference schemes for the $\mathrm{KdV}$ equation are absolutely unstable. Our results are in complete agreement with this conclusion.
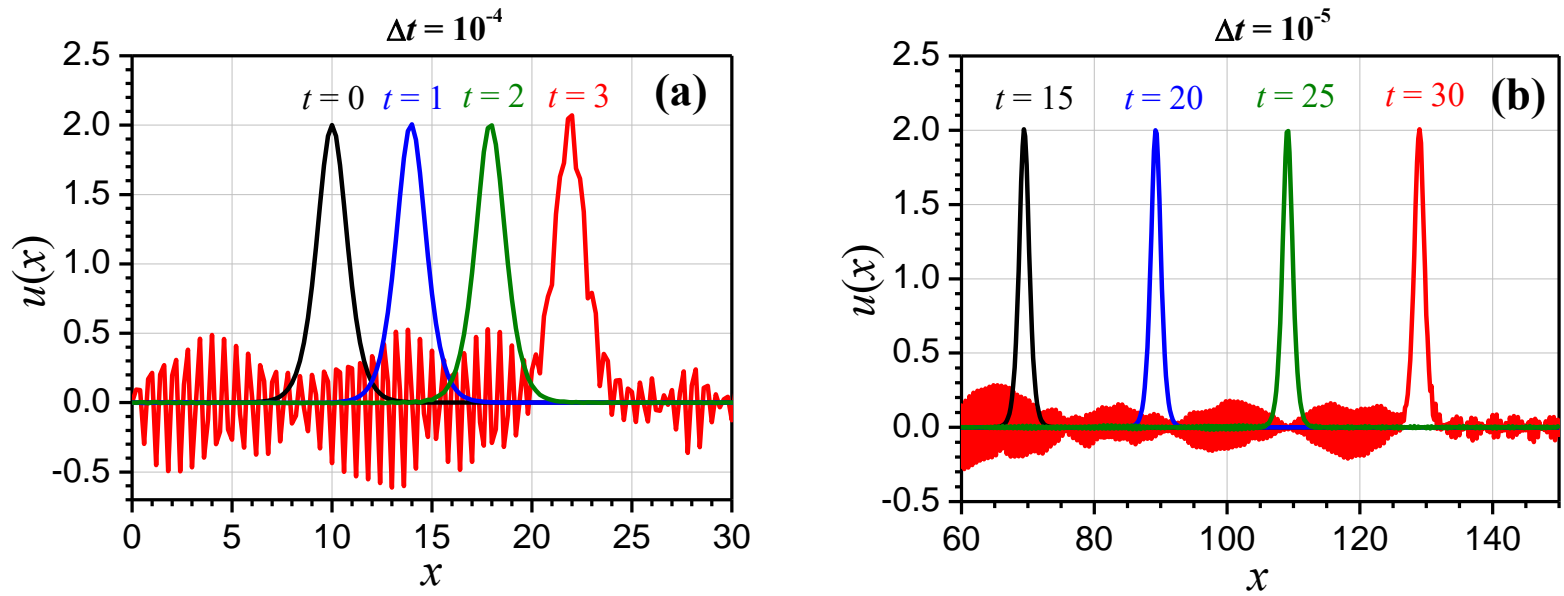

Fig .5. Numerical solution using an explicit difference scheme $(\sigma=0)$ with different integeration steps (by an order)

\section{ANALYTICAL STUDY}

When using the finite difference method, it is not the original partial differential equation that is solved numerically, but a modified equation called the differential approximation of the difference scheme [33-35]. The right side of this approximation is the approximation error and is equal to the difference between the original partial differential equation and its finitedifference analogue. Investigation of the right-hand sides of differential approximations makes it possible to establish the predominant contribution to the approximation error of the highest derivatives and the related properties of difference schemes such as dissipation and dispersion. It is known that if the main term in the expression for the approximation error contains derivatives of an even order, then the dominant properties of difference schemes will be dissipative, and if derivatives of an odd order, then the dominant properties will be dispersive.

Let us analyze the family of schemes (5) using the method of the modified equation [3335]. To do this, first we replace the sought function $u(x, t)$ by $f(x, t)=\alpha \cdot u(x, t)$. This allows one to get rid of the coefficient $\alpha$ as in both the original equation

$$
\frac{\partial f}{\partial t}+\frac{\partial}{\partial x}\left(\frac{f^{2}}{2}\right)+\beta \frac{\partial^{3} f}{\partial x^{3}}=0
$$

and in the finite-difference approximation

$$
\frac{f_{m}^{k+1}-f_{m}^{k}}{\Delta t}+\frac{1}{\Delta x}\left(\frac{\left(f_{m+1}^{k+\sigma}+f_{m}^{k+\sigma}\right)^{2}}{8}-\frac{\left(f_{m}^{k+\sigma}+f_{m-1}^{k+\sigma}\right)^{2}}{8}\right)+\beta \frac{f_{m+2}^{k+\sigma}-2 f_{m+1}^{k+\sigma}+2 f_{m-1}^{k+\sigma}-f_{m-2}^{k+\sigma}}{2 \Delta x^{3}}=0
$$


That is, the factor $\alpha$ is simply the scaling factor for the ordinate.
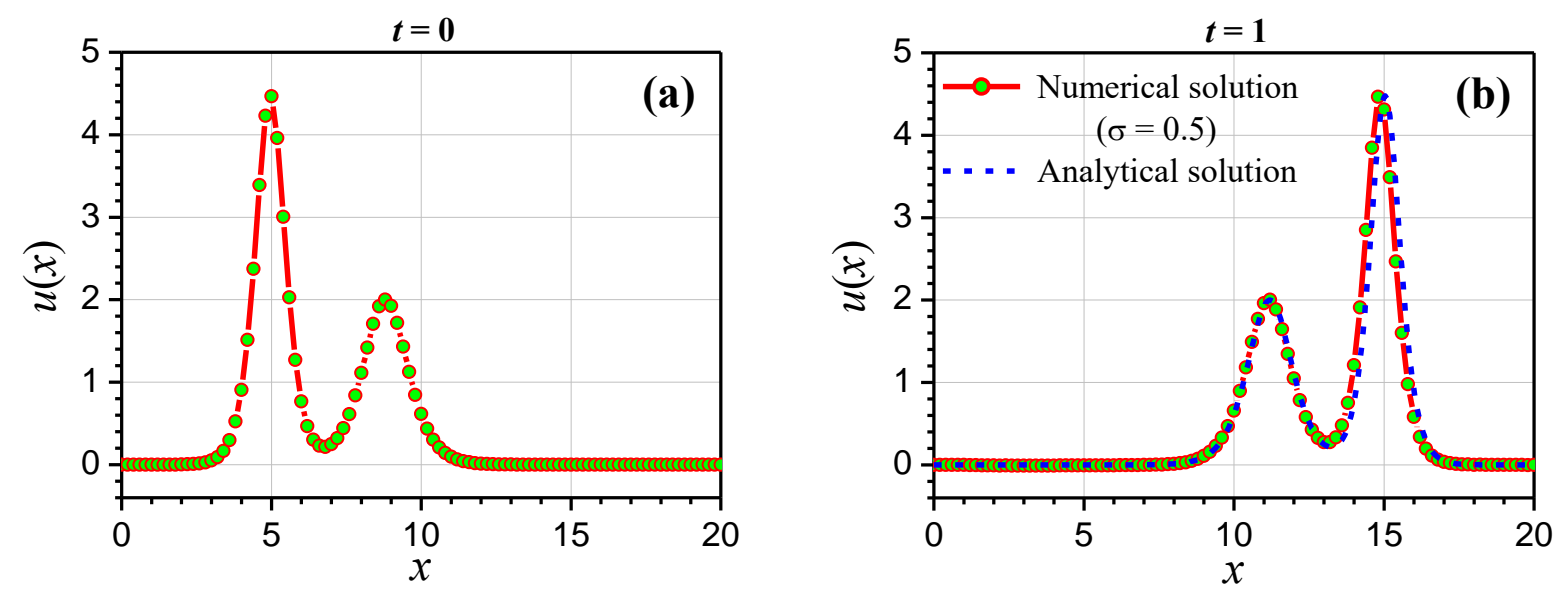

Fig.6. Numerical solution of the problem of collision of solitons: (a) initial condition, (b) comparison with the analytical solution at the time $\mathrm{t}=1$.

Next, we expand the two-dimensional function $f(x, t)$ in a Taylor series at a point $\left(x_{m}, t^{k+1 / 2}\right)$ and substitute it into scheme (7). Leaving the terms in the resulting expression not higher than the second order of smallness, we can write

$$
\begin{aligned}
& f_{t}^{\prime}+f \cdot f_{x}^{\prime}+\beta f_{x x x}^{\prime \prime \prime}=-\left[f \cdot f_{x t}^{(\mathrm{II})}+f_{t}^{\prime} f_{x}^{\prime}+\beta f_{x x x t}^{(\mathrm{IV})}\left(\sigma-\frac{1}{2}\right) \Delta t-\right. \\
& -\left[\frac{1}{8} f \cdot f_{x t t}^{(\mathrm{III})}+f_{t}^{\prime} f_{x t}^{(\mathrm{II})}\left(\sigma-\frac{1}{2}\right)^{2}+\frac{1}{8} f_{t t}^{\prime \prime} f_{x}^{\prime}+\frac{f_{t t t}^{\prime \prime \prime}}{24}+\frac{\beta}{8} f_{x x x t t}^{(\mathrm{V})}\right] \Delta t^{2}-\left[\frac{2}{3} f \cdot f_{x x x}^{\prime \prime \prime}+f_{x}^{\prime} f_{x x}^{\prime \prime}+\beta f_{x x x x x}^{(\mathrm{V})}\right] \frac{\Delta x^{2}}{4}
\end{aligned}
$$

In this expression (8), to simplify notation, the indices $m$ and $k+1 / 2$ for the function $f$ and all its derivatives are omitted. We focus our attention on the first-order term in $\Delta t$. Using the original equation (6), we get rid of the time derivatives:

$$
\begin{aligned}
& f_{t}^{\prime}=-f \cdot f_{x}^{\prime}-\beta f_{x x x}^{\prime \prime \prime} \\
& f_{x t}^{\prime \prime}=\frac{\partial}{\partial x}\left(-f \cdot f_{x}^{\prime}-\beta f_{x x x}^{\prime \prime \prime}\right)=-\left(f_{x}^{\prime}\right)^{2}-f \cdot f_{x x}^{\prime \prime}-\beta f_{x x x x}^{(\mathrm{IV})} \\
& f_{x x x t}^{(\mathrm{IV})}=-3\left(f_{x x}^{\prime \prime}\right)^{2}-4 f_{x}^{\prime} f_{x x x}^{\prime \prime \prime}-f \cdot f_{x x x x}^{(\mathrm{IV})}-\beta f_{x x x x x}^{(\mathrm{VI})}
\end{aligned}
$$

In addition, for simplicity, we use the boundedness of the function $f$ and all its derivatives in the domain of definition, and we replace the coefficients of the second-order terms by the constants $K_{l} K_{2}$. As a result, we finally get:

$$
\begin{aligned}
& f_{t}^{\prime}+f \cdot f_{x}^{\prime}+\beta f_{x x x}^{\prime \prime \prime}=K_{1} \Delta x^{2}+K_{2} \Delta t^{2}+ \\
& +\Delta t\left(\sigma-\frac{1}{2}\right)\left[\left\{f^{2} f_{x x}^{\prime \prime}+2 \beta f \cdot f_{x x x x}^{(\mathrm{IV})}+\beta^{2} f_{x x x x x x}^{(\mathrm{VI})}+3 \beta\left(f_{x x}^{\prime \prime}\right)^{2}\right\}+\left\{2 f \cdot\left(f_{x}^{\prime}\right)^{2}+5 \beta f_{x}^{\prime} f_{x x x}^{\prime \prime \prime}\right\}\right]
\end{aligned}
$$

Now let's analyze the resulting expression (10). In the term of the 1st order with respect to $\Delta t$, the square brackets contain two groups of terms: in the 1st curly bracket there are even 
derivatives with respect to $\mathrm{x}$, in the 2 nd - odd ones. I.e. the 1 st curly bracket corresponds to the schematic viscosity, and the 2nd - to the schematic dispersion. In this case, the sign of the coefficient in front of the dispersion terms is insignificant, while in front of the diffusion terms it is very important. This sign is determined by the difference $(\sigma-1 / 2)$.

When $\sigma>1 / 2$, the coefficient in front of the first-order diffusion terms is positive, and scheme (7) implements equation (6) with an additional viscosity proportional to the first power of the time step. This provides, on the one hand, stable behavior in the calculation process, on the other hand, distortion of the solution; over time, the initial perturbation is smeared out. It is this effect that we observed in the numerical solution using a completely implicit scheme (see Fig. 3).

When $\sigma<1 / 2$, the coefficient in front of the first-order diffusion terms is negative. That is, equation (10) gets negative viscosity. This means that scheme (7) becomes absolutely unstable. And since the absolute value of the coefficient is still proportional to the 1st power of the time step, the destruction of the solution occurs the earlier, the larger the time step is. This is precisely what we observed when experimenting with an explicit difference scheme (see Fig. 5).

In addition, now the assertion of theoretical works $[24,25]$ about the absolute instability of explicit two-layer difference schemes for the $\mathrm{KdV}$ equation can be extended for the family of schemes (7): all schemes (7) are absolutely unstable for $\sigma<1 / 2$, i.e. with "any prevalence of explicitness".

The highlighted value of $\sigma$ is $1 / 2$. For this single value, the first-order term in (10) vanishes, and thus scheme (7) receives the second-order approximation in both variables, $O\left(\Delta t^{2}+\Delta x^{2}\right)$. In addition, the effects of the scheme viscosity and 1st order dispersion are nullified. It is precisely because of this that, in a numerical solution on a somewhat coarse grid, it was possible to obtain the transport of a soliton with practically no distortions over considerable distances (see Fig. 2).

So, scheme (5) with $\sigma=1 / 2$ showed the best results in modeling of the problem of the soliton transfer.

\section{VERIFICATION OF THE APPROXIMATION ORDER}

Using the example of the problem of collision of solitons, we numerically verify the order of approximation of scheme (5) at $\sigma=0.5$. For equation (1) with parameters $\alpha=6, \beta=1$, there is the following analytical solution to this problem [36]:

$$
U_{\text {exact }}(x, t)=\frac{22.5 \operatorname{ch}[x-4 t-8]^{2}+10 \operatorname{sh}[1.5(x-9 t-5.5)]^{2}}{\{\operatorname{ch}[1.5(x-9 t-5.5)] \operatorname{ch}[x-4 t-8]+2 \operatorname{ch}[0.5(x-19 t-0.5)]\}^{2}}
$$

We will use this solution to set the initial condition (Fig.6a) and then estimate the error of the numerical solution. For this, we use the following definitions of the error of the numerical solution at the time $t^{k}$ :

$$
\begin{aligned}
& \text { 1) } \varepsilon_{L 2}=\sqrt{\frac{1}{M+1} \sum_{m=0}^{M}\left[u_{m}^{k}-U_{\text {exact }}\left(x_{m}, t^{k}\right)\right]^{2}} \\
& \text { 2) } \varepsilon_{C}=\max _{0 \leq m \leq M}\left(\mid u_{m}^{k}-U_{\text {exact }}\left(x_{m}, t^{k}\right)\right)
\end{aligned}
$$


At the first stage, we choose the size of the spatial step $\Delta x_{(I)}=0.2$, as in the previous problem on the transfer of a soliton. We solve the problem in the computational domain $x \in[0,20]$ in the time interval $t \in[0,1]$ (Fig.6b) with automatic selection of the time step in order to determine the maximum allowable time step. It turned out $\Delta t_{\max } \approx 4 \cdot 10^{-3}$. Then we make calculations by disabling the automatic selection mechanism and decreasing the time step until the errors at the end of the calculation $t_{\text {end }}=1$, calculated by formulas (12), stop decreasing. This means that in the approximation error

$$
\varepsilon(\Delta t, \Delta x)=E_{\Delta t}(\Delta t)+E_{\Delta x}(\Delta x)
$$

the part related to the time step became negligible compared to the part related to the space step: $E_{\Delta t}\left(\Delta t_{0}\right)<<E_{\Delta x}\left(\Delta x_{(I)}\right)$. It turned out for our problem $\Delta t_{0} \approx 2 \cdot 10^{-6}$. Wherein

$$
\begin{aligned}
& \text { 1) } E_{L 2}\left(\Delta x_{(I)}\right)=0.21359560124 \\
& \text { 2) } E_{C}\left(\Delta x_{(I)}\right)=0.89613301323
\end{aligned}
$$

We do the calculation again, leaving the step $\Delta t_{0}$ unchanged, but reducing the step in space by 2 times:. We get

$$
\begin{aligned}
& \text { 1) } E_{L 2}\left(\Delta x_{(I)} / 2\right)=0.05356903839 \\
& \text { 2) } E_{C}\left(\Delta x_{(I)} / 2\right)=0.22397163990
\end{aligned}
$$

Thus, with good accuracy

$$
\frac{E_{L 2}\left(\Delta x_{(I)}\right)}{E_{L 2}\left(\Delta x_{(I)} / 2\right)}=3.99, \frac{E_{C}\left(\Delta x_{(I)}\right)}{E_{C}\left(\Delta x_{(I)} / 2\right)}=4.00
$$

That is, with a 2-fold decrease in the spatial step, the associated approximation error decreased by a factor of 4 . This means that the scheme has a 2 nd order of approximation in space and an error $O\left(\Delta x^{2}\right)$.

To determine the order of approximation in time, we find the ratio:

$$
\frac{E_{\Delta t}\left(\Delta t_{\max }\right)}{E_{\Delta t}\left(\Delta t_{\max } / 2\right)}=\frac{\varepsilon\left(\Delta t_{\max }, \Delta x_{(I)}\right)-E_{\Delta x}\left(\Delta x_{(I)}\right)}{\varepsilon\left(\Delta t_{\max } / 2, \Delta x_{(I)}\right)-E_{\Delta x}\left(\Delta x_{(I)}\right)}
$$

As the spatial part of the error $E_{\Delta x}\left(\Delta x_{(I)}\right)$, we can use the previously obtained values (14). Let us calculate the total error again:

$$
\begin{aligned}
& \text { 1) } \varepsilon_{L 2}\left(\Delta t_{\max }, \Delta x_{(I)}\right)=0.21931557047, \varepsilon_{L 2}\left(\Delta t_{\max } / 2, \Delta x_{(I)}\right)=0.2150291787 \\
& \text { 2) } \varepsilon_{C}\left(\Delta t_{\max }, \Delta x_{(I)}\right)=0.91958522470, \varepsilon_{C}\left(\Delta t_{\max } / 2, \Delta x_{(I)}\right)=0.90208673036
\end{aligned}
$$

Substituting all values into (15) we obtain

$$
\frac{E_{L 2}\left(\Delta t_{\max }\right)}{E_{L 2}\left(\Delta t_{\max } / 2\right)}=3.99, \frac{E_{C}\left(\Delta t_{\max }\right)}{E_{C}\left(\Delta t_{\max } / 2\right)}=3.94
$$


This means that the scheme has a 2nd order of approximation in time and an error $O\left(\Delta t^{2}\right)$.

\section{CONCLUSIONS}

In this paper, we investigated a family of weighted two-layer difference schemes for the Korteweg-de Vries equation on an Eulerian difference grid.

It is shown numerically that the best results are obtained using an explicit-implicit difference scheme of the Crank-Nichols type of the second order of approximation $O\left(\Delta t^{2}+\Delta x^{2}\right)$. This scheme is capable of stably reproducing a stationary solution with good accuracy for a long time. The second order of approximation in both variables is numerically confirmed by the example of the problem of collision of solitons.

A completely implicit two-layer scheme of the 1st order in time and 2nd in space $O\left(\Delta t+\Delta x^{2}\right)$, although absolutely stable, nevertheless, due to the high scheme viscosity, significantly distorts the solution.

The calculation with the use of an explicit two-layer scheme has never been completed. There always came a moment of loss of stability, even with a very small time step. Although, up to this point, the solution was quite acceptable.

An analytical study of the family of finite-difference schemes (5) using the modified equation method fully confirmed the results of numerical experiments. The analysis of the structure of the approximation error for a family of two-layer finite-difference schemes made it possible to explicitly show the reasons for the success of explicitly implicit Crank- Nichols type schemes with $O\left(\Delta t+\Delta x^{2}\right)$ and the absolute instability of the family of schemes (5) in the case of "prevalence of explicitness" with a parameter $\sigma<0.5$. High scheme viscosity of absolutely stable fully implicit two-layer schemes of the 1st order $O\left(\Delta t+\Delta x^{2}\right)$ indicate the need to improve the accuracy of the space-time approximation.

An important advantage of the considered schemes is their simplicity and transparency of the basic mathematical constructions.

\section{REFERENCES}

[1] R.Kh. Zeytounian, "Nonlinear long waves on water and solitons", Phys. Usp., 38 (12), 13331381 (1995).

[2] J. Boussinesq, "Théorie de l'intumescence liquide appelée onde solitaire ou de translation se propageant dans un canal rectangulaire", Comptes Rendus l'Académie des Sci, 72, 755-759 (1871).

[3] J.M. Burgers, "A mathematical model illustrating the theory of turbulence", Adv. Appl. Mech., 1, 171-199 (1948).

[4] J.S. Russell, "Report on Waves", Report of the 14th meeting of the British Association for the Advancement of Science, York, September 1844, London, 1845, 311-390 (1844-1845).

[5] D. J. Korteweq and G. de-Vries, "On the change of form of long waves achancing in a rectangular canal, and on a new type of long stationary waves", Philos. Mag., 39, 422-443 (1895).

[6] N.J. Zabusky and M.D. Kruskal, "Interaction of "solitons" in a collisionless plasma and the recurrence of initial states", Phys. Rev. Lett., 15, 240-243 (1965). 
[7] C.H. Su and C.S. Gardner, "Korteweg-de Vries equation and generalizations: III. Derivation of the Korteweg- de Vries equation and Burgers equation", J. Math. Phys., 10, 536-539 (1969).

[8] Y. Kivshar, "Dark optical solitons with reverse-sign amplitude", Phys. Rev. A, 44, R1446-9 (1991).

[9] Y. A. Berezin, V. I. Karpman, "Nonlinear evolution of disturbances in plasma and other dispersive media", Soviet Physics JETP, 24, 1049-1056 (1967).

[10] A. V. Gurevich and L. P. Pitaevski, "Nonstationary structure of a collision less shock wave", Zh. Eksp. Teor. Fiz., 65, 590-604 (1973).

[11] Yu.S. Kivshar, G.P. Agrawal, Optical Solitons: From Fibers to Photonic Crystals. 2nd ed., Academic Press, (2008).

[12] R.K. Dodd, J.C. Eilbeck, J.D. Gibbon and H.C. Morris, Solitons and Nonlinear Wave Equations, London: Academic, (1982).

[13] G.B. Whitham, Linear and Nonlinear Waves, Wiley, New York, NY (1974).

[14] N. Yi, Y. Huang, and H. Liu, "A direct discontinuous Galerkin method for the generalized Korteweg-de Vries equation: Energy conservation and boundary effect", $J$. Comput. Phys., 242, 351-366 (2013).

[15] J. Bona, H. Chen, O. Karakashian, and Y. Xing, "Conservative, discontinuousGalerkin methods for the generalized Korteweg-de Vries equation", Mathematics of Computation, 82(283), 1401-1432 (2013). https://www.jstor.org/stable/42002703

[16] Q. Zhang, Y. Xia, "Conservative and Dissipative Local Discontinuous Galerkin Methods for Korteweg-de Vries Type Equations", Commun. Comput. Phys., 25(2), 532-563 (2019). doi: 10.4208/cicp.OA-2017-0204

[17] H. Kalisch and X. Raynaud, "On the rate of convergence of a collocation projection of the KdV equation," Mathematical Modelling and Numerical Analysis, 41(1), 95-110 (2007).

[18] Turabi Geyikli, "Collocation Method for Solving the Generalized KdV Equation", J.Appl. Math. Phys., 8, 1123-1134 (2020). https://www.scirp.org/journal/jamp.

[19] Anna Karczewska, Piotr Rozmej, Maciej Szczeciński, Bartosz Boguniewicz, "A Finite Element Method for Extended KdV Equations", Int. J. Appl. Math. Comput. Sci., 26(3), 555-567 (2016). https://doi.org/10.1515/amcs-2016-0039

[20] A. Karczewska, M. Szczeciński, P. Rozmej, and B. Boguniewicz, "Finite element method for stochastic extended KdV equations", Computational Methods in Science and Technology, 22(1), 19-29 (2016).

[21] N.K. Amein, M.A. Ramadan, "A small time solutions for the KdV equation using Bubnov-Galerkin finite element method", J. Egyptian Math. Society, 19(3), 118-125 (2011). https://doi.org/10.1016/j.joems.2011.10.005

[22] A.C. Vliegenthart, "On finite-difference methods for the Korteweg-de Vries equation", J Eng Math, 5, 137-155 (1971). https://doi.org/10.1007/BF01535405

[23] K. Goda, "On stability of some finite difference schemes for the korteweg-de vries equation", Journal of the Physics Society of Japan, 39(1), 229-236 (1975). http://dx.doi.org/10.1143/JPSJ.39.229

[24] A.A. Samarskii, V/I/ Mazhukin, P.P. Matus, I.A. Mikhailyuk, "L2-Konservativny`e skhemy` dlia uravneniia Kortevega-de Frisa”, Doclady`Akademii Nauk, 357(4), 458-461 (1997).

[25] V.I. Mazhukin, P.P.Matus, I.A. Mikhailyuk, "Finite-difference schemes for the korteweg—de vries equation", Differential Equations, 36(5), 789-797 (2000). doi:10.1007/bf02754240 
[26] V.M. Goloviznin, S.A. Karabasov, D.A. Sukhodulov, "Variatcionny 'i podhod k polucheniiu raznostnoi` skhemy`s prostranstvenno rasshcheplennoi` vremennoi` proizvodnoi` dlia uravneniia Kortvega - de Vriza”, Matematicheskoe modelirovanie, 12 (4), (2000).

[27] S.H. Zhu, "A scheme with a higher-order discrete invariant for the KdV equation", Appl. Math. Let., 14(1), 17-20 (2001).

[28] Wang Hui-Ping, Wang Yu-Shun, and Hu Ying-Ying, "An Explicit Scheme for the KdV Equation", Chinese Phys. Lett., 25, 2335 (2008). DOI: https://doi.org/10.1088/0256307X/25/7/002

[29] H. Holden, U. Koley, N.H. Risebro, "Convergence of a fully discrete finite difference scheme for the Korteweg-de Vries equation", IMA Journal of Numerical Analysis, 35(3), 1047-1077 (2014). doi:10.1093/imanum/dru040

[30] Olusola Kolebaje, Emmanuel Oluwole Oyewande, "Numerical Solution of the Korteweg De Vries Equation by Finite Difference and Adomian Decomposition Method", International Journal of Basic and Applied Sciences, 1(3), (2012). DOI: $10.14419 /$ ijbas.v1i3.131.

[31] G. D. Akrivis, "Finite difference discretization of the cubic Schrödinger equation", IMA Journal of Numerical Analysis, 13(1), 115-124 (1993). doi:10.1093/imanum/13.1.115

[32] Georgios Akrivis, Vassilios A. Dougalis, Ohannes Karakashian, "Solving the systems of equations arising in the discretization of some nonlinear P.D.E.'s by implicit Runge-Kutta methods", ESAIM Mathematical Modelling and Numerical Analysis - Modélisation Mathématique et Analyse Numérique, 31(2), 251-287 (1997).

[33] R.E. Warming, B.J. Hyett, "The Modified Equation Approach to the Stability and Accuracy Analysis of finite-difference Methods", J. Comput. Phys., 14, 159-179 (1974).

[34] Yu.I. Shokin, "Pervoe differentcial noe priblizhenie", Novosibirsk: Nauka, (1979).

[35] D. Anderson, J. Tannehill, R. Pletcher, Computational Fluid Mechanics and Heat Transfer, New York, Hemisphere Publishing Corporation, (1984).

[36] Yong Duan, Zhang Peng, Zeng Yan, Ahmed Naji, "Convergence estimate of Cauchy problems for the shallow water equations with MQ quasi-interpolation", Preprint, Sep., (2019). DOI: 10.13140/RG.2.2.17814.50244

Received October 10, 2020 